\title{
THE ARMENDARIZ GRAPH OF A RING
}

\author{
Cinat AbDíŏ̆LU ${ }^{1}$ \\ Department of Primary Education \\ Karamanoğlu Mehmetbey University \\ Yunus Emre Campus, 70100, Karaman, Turkey \\ e-mail: cabdioglu@kmu.edu.tr \\ Ece Yetkin Çelikel \\ Department of Mathematics \\ Gaziantep University, Gaziantep, Turkey \\ e-mail: yetkinece@gmail.com
}

AND

Angsuman Das

Department of Mathematics

Presidency University, Kolkata, India

e-mail: angsumandas054@gmail.com

\begin{abstract}
In this paper we initiate the study of Armendariz graph of a commutative ring $R$ and investigate the basic properties of this graph such as diameter, girth, domination number, etc. The Armendariz graph of a ring $R$, denoted by $A(R)$, is an undirected graph with nonzero zero-divisors of $R[x]$ (i.e., $\left.Z(R[x])^{*}\right)$ as the vertex set, and two distinct vertices $f(x)=\sum_{i=0}^{n} a_{i} x^{i}$ and $g(x)=\sum_{j=0}^{m} b_{j} x^{j}$ are adjacent if and only if $a_{i} b_{j}=0$, for all $i, j$. It is shown that $A(R)$, a subgraph of $\Gamma(R[x])$, the zero divisor graph of the polynomial ring $R[x]$, have many graph properties in common with $\Gamma(R[x])$.
\end{abstract}

Keywords: Armendariz property, diameter, girth, zero-divisor graph.

2010 Mathematics Subject Classification: 05C12, 05C25.

\footnotetext{
${ }^{1}$ Corresponding author.
} 


\section{REFERENCES}

[1] D.F. Anderson and P.S. Livingston, The zero-divisor graph of a commutative ring, J. Algebra 217 (1999) 434-447. doi:10.1006/jabr.1998.7840

[2] M. Axtell, J. Coykendall and J. Stickles, Zero-divisor graphs of polynomials and power series over commutative rings, Commun. Algebra 33 (2005) 2043-2050. doi:10.1081/AGB-200063357

[3] I. Beck, Coloring of commutative rings, J. Algebra 116 (1988) 208-226. doi:10.1016/0021-8693(88)90202-5

[4] B. Bollobás, Modern Graph Theory, Graduate Texts in Mathematics (New York, Springer-Verlag, 1998). doi:10.1007/978-1-4612-0619-4

[5] T.W. Hungerford, Algebra. Graduate Texts in Mathematics 73 (New York, Springer-Verlag, 1974).

[6] I. Kaplansky, Commutative Rings (rev. ed. Chicago, Univ. of Chicago Press, 1974).

[7] T.G. Lucas, The diameter of a zero-divisor graph, J. Algebra 301 (2006) 174-193. doi:10.1016/j.jalgebra.2006.01.019

[8] N.H. McCoy, (1942), Remarks on divisors of zero, Amer. Math. Monthly 49 (1942) 286-295. doi: $10.2307 / 2303094$

[9] M.B. Rege and S. Chhawchharia, Armendariz rings, Proc. Japan Acad. Ser. A Math. Sci. 73 (19970 14-17. doi:10.3792/pjaa.73.14

Received 13 November 2017 Revised 30 May 2018 Accepted 4 June 2018 